\title{
CALR Protein Variant
}

National Cancer Institute

\section{Source}

National Cancer Institute. CALR Protein Variant. NCI Thesaurus. Code C129836.

A variation in the amino acid sequence for the calreticulin protein. 